Kardiologe 2009 [Suppl 1] · 3:3

DOI 10.1007/s12181-009-0187-0

(c) Springer Medizin Verlag 2009

\section{T. Meinertz}

Klinik und Poliklinik für Kardiologie/Angiologie, Universitäres Herzzentrum Hamburg

\title{
Vorwort
}

Physiologisch betrachtet steigt mit der Herzfrequenz auch der Sauerstoff- und Energiebedarf des Herzmuskels, ein Umstand, der insbesondere bei der Koronaren Herzkrankheit eine wichtige Rolle spielt. Patienten mit koronarer Herzerkrankung profitieren deshalb besonders von einer Frequenzreduktion und der hierdurch verlängerten diastolischen Perfusionszeit. Üblicherweise werden hierzu Betablocker eingesetzt. Diese Therapie wurde bisher erfolgreich bei einer Vielzahl von Patienten durchgeführt, allerdings haben Betarezeptoren mehrere Funktionen und entsprechend ist eine Betablockade kein selektiver Therapieansatz für die Herzfrequenzsenkung.

Früher gab es für Betarezeptorenblocker keine therapeutischen Alternativen. Seit wenigen Jahren steht für Patienten, die Betablocker nicht tolerieren, bzw. Kontraindikationen aufweisen, ein neues Medikament als Alternative zur Verfügung. Es reduziert ebenso wie Betarezeptorenblocker die Herzfrequenz ohne andere kardiovaskuläre Funktionen zu beeinflussen. Dieses Medikament (Ivabradin) gehört einer neu entdeckten Substanzklasse (Hemmstoffe des sogenannten $I_{\mathrm{f}}$-Kanals) an. Es setzt selektiv die Entladungsfrequenz des Sinusknotens und damit die Herzfrequenz herab.

Schon zur Zulassung konnte in einem groß angelegten Untersuchungsprogramm nachgewiesen werden, dass Ivabradin bei stabiler Angina pectoris genauso wirksam ist, wie Betarezeptorenblocker. Es verbessert die körperliche Belastbarkeit und senkt die Ischämieschwelle in der gleichen Größenordnung, wie Betarezeptorenblocker. Für die letztgenannten Substanzen typische $\mathrm{Ne}$ benwirkungen wurden dagegen nicht beobachtet. In diesen Studien konnte außerdem nachgewiesen werden, dass Ivabradin erfolgreich mit anderen antiischämisch wirksamen Medikamenten - z.B. Kalziumanta- gonisten vom Dihydropyridintyp - kombiniert werden kann.

Es war daher nur konsequent, Ivabradin auch in die Leitlinien zur Therapie der stabilen Angina pectoris (Leitlinien der Europäischen Gesellschaft für Kardiologie) aufzunehmen, um die therapeutische Zielfrequenz von 55-60 Schlägen/Minute zu erreichen.

Während bei Patienten mit normaler linksventrikulärer Funktion eine solche Herzfrequenzeinstellung durch Betarezeptorenblocker oder Ivabradin meist ohne Schwierigkeiten erreichbar ist, machen $\mathrm{Pa}-$ tienten mit deutlich eingeschränkter linksventrikulärer Funktion diesbezüglich Probleme: Die Ausgangsherzfrequenz ist meist deutlich höher und eine rasche Absenkung der Herzfrequenz durch Betarezeptorenblocker wird auch hämodynamisch nicht gut vom Patienten toleriert. Es stellten sich dementsprechend folgende Fragen:

1. Lassen sich bei Patienten mit koronarer Herzkrankheit und deutlich eingeschränkter linksventrikulärer Funktion (LVEF <40\%) durch Ivabradin günstige klinische Wirkungen (Verminderung der Sterblichkeit, seltener stationäre Aufnahmen wegen eines Herzinfarktes, seltener stationäre Aufnahmen wegen einer Herzinsuffizienz, weniger interventionelle Maßnahmen) erreichen?

2. Wie ist die Abhängigkeit einer derartigen Wirkung von der Ausgangsfrequenz? Profitieren alle Patienten unabhängig von der Ausgangsfrequenz oder ist der therapeutische Nutzen nur ab einer gewissen (erhöhten!) Herzfrequenz vorhanden?

3. Lassen sich bei diesen Patienten Betarezeptorenblocker und Ivabradin kombinieren? Kann z.B. immer dann, wenn durch Betarezeptorenblocker eine adäquate Herzfrequenzsenkung nicht erreichbar ist, zur optimalen Herzfrequenzsenkung Ivabradin zusätzlich verabreicht werden?

Diese Fragen sollten durch die Ergebnisse einer kürzlich abgeschlossenen und publizierten Studie (BEAUTI $I_{\mathrm{f}} \mathrm{UL}$-Studie) beantwortet werden. Die Ergebnisse dieser Studie werden im vorliegenden Journal noch einmal referiert und zusammengefasst. Sie lassen sich in ihrer Komplexität nur verstehen, wenn sie nicht nur im Detail analysiert, sondern auch in dem größeren Zusammenhang koronare Herzkrankheit, reduzierte linksventrikuläre Funktion, Herzinsuffizienz, optimaler Herzfrequenzbereich und Betablockermedikation gestellt werden.

Folgendes ist nach dem Ergebnis der BEAUTI $I_{\mathrm{f}} \mathrm{UL}-$ Studie jedoch sicher:

a. Die Herzfrequenz ist ein eigenständiger Risikomarker

b. Betarezeptorenblocker können mit Ivabradin kombiniert werden.

c. Auch unter Langzeitbedingungen und bei kranken Patienten ist die $\mathrm{Ne}$ benwirkungshäufigkeit von Ivabradin gering.

d. Betarezeptorenblocker gehören auch weiterhin zur Standardmedikation der Patienten mit eingeschränkter linksventrikulärer Funktion.

e. Ivabradin kann (soll?) immer dann verabreicht werden, wenn sich die Herzfrequenz durch Betarezeptorenblocker bei diesen Patienten nicht auf unter $70 / \mathrm{min}$. senken lässt.

\section{Korrespondenzadresse \\ Prof. Dr. med. Thomas Meinertz}

Direktor der Klinik und Poliklinik für Kardiologie/ Angiologie

Universitäres Herzzentrum Hamburg

Martinistraße 52

20246 Hamburg 